\title{
Genital herpes and encephalitis; a case history
}

\author{
M. Pathiraja, N. Abegunasekara, A. Gunapala
}

\begin{abstract}
A 19-year-old, previously healthy unmarried girl presented with sudden onset loss of consciousness preceded by genital ulcers, fever and headache. Examination revealed mild neck stiffness, 4/15 of GCS, and upper motor neuron signs in all four limbs. In addition, there were multiple, superficial and tender genital ulcers. Electro Encephalogram (EEG) showed evidence of encephalitis and subsequent serological tests revealed presence of HSV antibodies. Her condition improved remarkably following intravenous Aciclovir therapy. Presence of typical herpetic ulcers, positive type specific serology test for HSV, evidence of encephalitis in the EEG and good response to Aciclovir, favoured the possible diagnosis of Herpes Simplex Encephalitis (HSE).
\end{abstract}

Key words: Genital ulcers, Herpes simplex encephalitis (HSE), Aciclovir

\footnotetext{
Authors: corresponding author: M. Pathiraja, MBBS, PgD Ven, MD; Senior Registrar, National STD/AIDS Control Programme, No 29, De Saram Place, Colombo 10, Sri Lanka, Email: malathipathiraja@gmail.com

N Abeygunasekara, MBBS, PgD Ven, MD; Consultant Venereologist, Teaching Hospital, Kalubowila, Sri Lanka

A Gunapala, MBBS, MD; Consultant Physician, Teaching Hospital, Kalubowila, Sri Lanka

Acknowledgement: Dr Ajith Karawita, Consultant Venereologist, Teaching Hospital Anuradhapura

Conflict of interest: Authors claim no conflicts of interest

Funding: Self financing

Originality, previous publications or presentations: This is an original work and there have not been any previous publications

Submitted: 16.11.2015, Accepted: 26.11.2015
}

\section{Full article}

\section{Introduction}

Herpes simplex virus (HSV) infection is one of the commonest causes for genital ulcers (1). There are about 530 million people living with Herpes Simplex Virus (HSV 2) (2). In Sri Lanka, during the year 2013, HSV infection was the commonest STI reported with 2500 cases (3). Usually herpes infection resolves without any complications but rarely disseminates to other organs like liver, lungs and brain. Herpes simplex encephalitis (HSE) is a rare complication and reported in several parts of the world. However, a literature survey did not reveal any reported cases from Sri Lanka. In countries like Sri Lanka, with limited diagnostic facilities, management of these cases is a challenge. High degree of clinical suspicion with early commencement of antivirals is vital to prevent neurological sequel and death.
Following is a possible case of HSV encephalitis which was managed mainly on clinical grounds with successful results. Herpes simplex encephalitis is a rare but fatal complication of a common STI.

\section{Case history}

A 19-year-old, unmarried girl, who had multiple painful genital ulcers of 4-day duration, mild headache, low grade fever of one-day duration and sudden loss of consciousness had been admitted to Teaching Hospital, Kalubowila. Initial management was done at the medical ward and 3 days after hospital admission, she was referred to the STD clinic.

According to hospital records, her Glasgow Coma Scale (GCS) was 4/15, upper motor neuron signs were noted in all four limbs and mild neck stiffness was present at the initial examination. Her full blood count showed elevated white blood cell count $(11,300$ cells $/ \mu \mathrm{l})$ and ESR was $62 \mathrm{~mm} / \mathrm{hr}$. Blood 
sugar levels, liver and renal function tests were within normal range. Cerebrospinal Fluid (CSF) report had elevated proteins $(66.6 \mathrm{mg} / \mathrm{dl})$ but acid fast bacilli or leucocytes were absent. CSF culture and blood culture were sterile. Although it was planned to test HSV type specific PCR of CSF, it could not be done due to financial problems. The Electroencephalography (EEG) showed features of encephalitis and CT scan of brain (non-contrast) was normal. MRI was not available. She was initially treated with intravenous ceftazidime $2 \mathrm{~g}$ three times a day and intravenous Aciclovir 500mg three times a day. With this treatment her condition improved gradually.

On the $3^{\text {rd }}$ day she was assessed at the STD clinic. Still she was confused and history was obtained from the mother. According to mother there was no associated photophobia, drowsiness, change in behaviour or seizures before loss of consciousness. She never had oral or genital ulcers in the past. There was no known past history of sexual exposures.

Examination revealed, multiple, tender, soft and superficial vulval ulcers. However, there were no oral ulcers, blisters over the face, red eye, arthritis or any other skin lesions. Other system examination was unremarkable. Tzanck test for Giant cells and HSV culture was negative probably as the ulcers were old and already treated with aciclovir. Dark ground examination for Treponema pallidum was also negative. Both HSV-1 and HSV-2 IgG antibodies were present but HSV-1 and HSV-2 IgM antibodies were absent. VDRL, TPPA and HIV ELISA tests were negative.

One week after the admission patient was able to speak few words responsively and walked with support. Genital ulcers were completely healed. The patient was discharged from the ward after completing the intravenous aciclovir for 14 days. By that time, she was conscious and oriented but had slurring of speech, motor responses slow and poor memory. However, she was able to walk without any support. She attended the STD clinic twice after being discharged from the ward. First visit was one week after the discharge and second in one-month time. There was a remarkable improvement of her memory, verbal responses and motor activities. Thereafter, she defaulted.

\section{Discussion}

The clinical presentation of the patient was highly suggestive of encephalitis. Leading causes of encephalitis are viral infections such as Japanese encephalitis, rabies, dengue, HSV infection etc. Sometimes certain bacterial and parasitic infections also lead to encephalitis (4). However, presence of genital ulcers in the patient lead to consider new set of differential diagnoses like herpes viral infection due to HSV 1 or 2, syphilis, Behcet's disease and HIV.

EEG confirmed the presence of encephalitis. Normal blood sugar levels, liver and renal function tests as well as negative blood cultures excluded basic metabolic problems and septicaemia which also could lead to altered level of consciousness. Negative serology excluded syphilis and HIV infections while absence of other related clinical features made Behcet's disease unlikely. CSF analysis revealed absence of leucocytes and slightly elevated proteins with negative routine bacterial culture which were compatible with a viral infection. Unfortunately, HSV PCR testing of CSF which is the key diagnostic test to confirm HSE could not be performed.

When considering the diagnosis there are no pathognomonic clinical findings associated with HSE (5). Typical findings on presentation include; alteration of consciousness (97\%), fever (92\%), dysphasia (76\%) and ataxia (40\%) (6). Similar features were present in this patient as well. Presence of typical herpetic ulcers, positive HSV serology and good response to aciclovir therapy favoured the diagnosis of herpes simplex encephalitis in this patient.

Herpes simplex encephalitis is a rare disease with the incidence of 1 per 250,000 to 
500,000 persons per year. Approximately $33 \%$ of cases occur in patients under the age of 20 years and about $50 \%$ in those over 50 years (7). The severity of genital herpes and the likelihood of complications such as meningitis, encephalitis are also greater in women than in men (8).

Although HSV-1 is the commonest cause of encephalitis among who are older than three years, HSV-2 also can affect nervous system. In adults, HSV-2 usually causes uncomplicated genital herpes but occasional cases of neurological involvement are recognized, ranging from meningitis, which may be recurrent to radiculomyelitis and rarely encephalitis (9-12). In this case HSV culture became negative. However, HSV serology revealed the presence of HSV-1 and HSV-2 antibody (IgG) showing the evidence of previous exposure to both HSV types.

The EEG always shows abnormal patterns (characteristic delta slow rhythms and periodic discharges) in herpes simplex encephalitis (13). Our patient also had similar changes in the EEG.

If untreated, the mortality of HSE is greater than $70 \%$ with only $2.5 \%$ of survivors returning to normality (7). HSE is a neurologic emergency and is the most important neurologic sequel of HSV infection. Residual neurological impairments among survivors are significant and depend on the patient's age and neurologic status at the time of diagnosis. Patients who are comatose at diagnosis have a poor prognosis regardless of their age. In non-comatose patients, the prognosis is age related, with better outcomes occurring in patients younger than 30 years (14).

The well-established and universally accepted treatment for HSE occurring outside the newborn period is intravenous aciclovir (10 $\mathrm{mg} / \mathrm{kg}$ every eight hours) for 10 to 14 days (15-16). In our patient, intravenous aciclovir was commenced immediately after the admission to the ward and continued for 14 days.
There are some factors affect the prognosis of HSE. A study by Utley et al showed that patients with short period ( $<5$ days) between presentation and treatment had better cognitive outcomes (14). At present, the only parameter that can be modified to improve the prognosis was identified as early initiation of aciclovir (17). Patient age and level of consciousness at onset of therapy have been identified elsewhere as major determinants of prognosis (14).

This highlights the importance of commencing aciclovir therapy as soon as possible considering the clinical features without waiting for definitive diagnosis where confirmatory testing facilities for HSE are limited. For this patient, intravenous aciclovir therapy was commenced immediately after the admission. This would have been the main reason for immediate recovery.

Significant morbidity exists even among those who are treated. Neurologic outcomes in survivors treated with aciclovir vary; no deficits or mild deficits in $38 \%$ of patients, moderate deficits among $9 \%$ and severe deficits in $53 \%$ of patients. Anterograde memory often is impaired even after successful treatment of HSE. Retrograde memory, executive function, and language ability also may be impaired (14).

As the patient defaulted after one month we were unable to determine the long term neurological deficits in our patient.

\section{References}

1. Report on global sexually transmitted infection surveillance. WHO Document Production Services, Geneva, Switzerland: WHO Press; 2013. www.who.int/reproductivehealthpublications/rtis/ stissss-surveillance.../en/ (accessed 2.4.2015)

2. WHO Sexually Transmitted Infections fact sheet N110. WHO Document Production Services, Geneva, Switzerland: WHO Media Centre; Updated Nov.2013.

www.who.int/mediacenter/factsheets/fs110/en/ (accessed 2.4.2015).

3. Annual report. Sri Lanka: National STD/AIDS control programme;2013. http://www.aidscontrol.gov.lk (accessed 2.4.2015) 
4. Mayoclinicstaff. Encephalitis. http://www.mayoclinic.org /diseases-conditions/ encephalitis/basics/causes/con-20021917 (accessed 2.4.2015)

5. Whitley R J ,Cobbs GG et al. Diseases that mimic Herpes simplex encephalitis.Diagnosis, presentation and outcome, NIAD Collaborative Antiviral study group. JAMA. July 14 1989:262(2):234-9

6. Whitley R J, Soong S J. Linneman C et al. Herpes simplex encephalitis. Clinical Assessment JAMA. Jan 15 1982:247(3):317-20

7. Whitley R J, Gnann J W et al. Viral encephalitis: familiar infection and emerging pathogen. Lancet 2002; 359: 507-14

8. Corey L Adans H G Brown ZA etal. Genital Herpes Simplex Viral infection: Clinical manifestations, Course and Complications. Ann

9. Aurelius E, Johansson B, Skoldenberg B, et al. Encephalitis in immunocompetent patients due to herpes simplex virus type 1 or 2 as determined by type-specific polymerase chain reaction and antibody assays of cerebrospinal fluid. J Med Virol 1993; 39: 179-86.

10. Dennett C, Cleator GM, Klapper PE. HSV-1 and HSV2 in herpes simplex encephalitis: a study of sixtyfour cases in the United Kingdom. J Med Virol 1997; 53:1-3.
11. Corey L, Adams HG, Brown ZA, et al. Genital herpes simplex virus infections: clinical manifestations, course, and complications. Ann Intern Med 1983; 98:958-72.

12. Tedder DG, Ashley R, Tyler KL, et al. Herpes simplex virus infection as a cause of benign recurrent lymphocytic meningitis. Ann Intern Med1994; 121:334-8.

13. Lai CW, Gragasin M E Electroencephalography in herpes simplex encephalitis J Clin Neurophysiol. 1988 Jan; 5(1):87-103

14. Uttely T F .Ogenda J A, Gibb A et al. The long term neuropsychological outcomes of Herpes simplex encephalitis in a series of unselected survivors. Neuropsychiatry neuropsychological behavioural. July 1997:10(3):180-9

15. Skoldenberg B, Forsgren M, Alestig K, et al. Aciclovir versus vidarabinein in herpes simplex encephalitis. Lancet 1984; ii: 707-11.

16. Whitley RJ, Alford CA, Hirsch MS, et al. Vidarabine versus Aciclovir therapy in herpes simplex encephalitis. N Engl J Med 1986; 314: 144-9.

17. Rachials F, Wolff $M$ et al. Outcome of and prognostic factors for Herpes simplex encephalitis in adult patients. Results of a multicenter study clinical infectious diseases 2002; 35:254-260 http://cid.oxfoordjournals.org/by guest on November 16, 2014 